\title{
Historical Cohort
}

National Cancer Institute

\section{Source}

National Cancer Institute. Historical Cohort. NCI Thesaurus. Code C71548.

Individuals grouped together for a study based on events that have happened in the past. 